\title{
Electronic structure and ionicity of actinide oxides from first principles calculations
}

\author{
L. Petit $*$ and A. Svane \\ Department of Physics and Astronomy, Aarhus University, DK-8000 Aarhus C, Denmark \\ Z. Szotek and W. M. Temmerman \\ Daresbury Laboratory, Daresbury, Warrington WA4 4AD, UK \\ G. M. Stocks \\ Materials Science and Technology Division, Oak Ridge National Laboratory, Oak Ridge, Tennessee 37831, USA
}

(Dated: November 1, 2018)

\begin{abstract}
The ground state electronic structures of the actinide oxides $\mathrm{AO}, \mathrm{A}_{2} \mathrm{O}_{3}$ and $\mathrm{AO}_{2}(\mathrm{~A}=\mathrm{U}, \mathrm{Np}$, $\mathrm{Pu}, \mathrm{Am}, \mathrm{Cm}, \mathrm{Bk}, \mathrm{Cf}$ ) are determined from first-principles calculations, using the self-interaction corrected local spin-density (SIC-LSD) approximation. Emphasis is put on the degree of f-electron localization, which for $\mathrm{AO}_{2}$ and $\mathrm{A}_{2} \mathrm{O}_{3}$ is found to follow the stoichiometry, namely corresponding to $\mathrm{A}^{4+}$ ions in the dioxide and $\mathrm{A}^{3+}$ ions in the sesquioxides. In contrast, the $\mathrm{A}^{2+}$ ionic configuration is not favorable in the monoxides, which therefore become metallic. The energetics of the oxidation and reduction of the actinide dioxides is discussed, and it is found that the dioxide is the most stable oxide for the actinides from Np onwards. Our study reveals a strong link between preferred oxidation number and degree of localization which is confirmed by comparing to the ground state configurations of the corresponding lanthanide oxides. The ionic nature of the actinide oxides emerges from the fact that only those compounds will form where the calculated ground state valency agrees with the nominal valency expected from a simple charge counting.
\end{abstract}

\section{INTRODUCTION}

Actinide oxides play a dominant role in the nuclear fuel cycle. [1] For many years, uranium dioxide has been the main fuel component in commercial nuclear reactors. The "burning" of $\mathrm{UO}_{2}$ results in considerable amounts of $\mathrm{Np}$ and $\mathrm{Pu}$ isotopes, as well as smaller quantities of minor actinides such as Am, Cm, Bk and Cf. In original, "once through" reactors, the highly radioactive waste that is produced resulted in very troublesome long time storage requirements; particularly of $\mathrm{Pu}$. However, it was soon realized that the $\mathrm{Pu}$ could be reprocessed from the spent fuel and used as alternative fuel in a new generation of reactors. The environmental and energy production issues apart, the fact that $\mathrm{Pu}$ is obtained from the decommisioning of nuclear weapons is yet another important consideration concerning its use as nuclear fuel. A mixture of $\mathrm{UO}_{2}$ and $\mathrm{PuO}_{2}$, the so-called mixed oxide (MOX), where $\mathrm{Pu}$ is blended with either natural or depleted Uranium, constitutes the preferred, $\mathrm{Pu}$ containing, fuel in existing nuclear reactors. Lately, $\mathrm{Np}$, as well as the minor actinides that accumulate during nuclear reactor operation, are also being considered for reprocessing. [2] Once separated from the spent fuel, these actinides can either be incorporated in durable ceramic waste for safe long time storage (and possible later recovery) or transmuted from long-lived isotopes to less radiotoxic short-lived isotopes through irradiation, thus taking part in the fuel cycle and reducing the long term nuclear waste management problem. Again oxides are being considered both with respect

*Electronic address: lpetit@phys.au.dk to the materials being used as fission/transmutation targets $\left(\mathrm{AO}_{2}\right)$ [3] and for the direct storage in the shape of durable ceramic glasses $\left(\mathrm{AO}_{2}, \mathrm{~A}_{2} \mathrm{O}_{3}\right)$. [2] For example $\mathrm{PuO}_{2}$ has long been the compound of choice for depositing $\mathrm{Pu}$ in long time repositories, given the observed stability with respect to oxidation. [4]

Regarding their behaviour under both reactor operation and storage conditions, it is crucial to understand the thermochemistry, thermophysics, and materials science of the actinide compounds. Given the toxicity of the materials involved, computer simulations, such as thermodynamic modelling [5] or, as in the present paper, electronic structure calculations can provide fundamental insights at a level not achieveable through experiment alone. Here we wish to focus specifically on the $f$-electrons, their contribution to the groundstate electronic properties of the actinide oxides, the role they play with regards to stability towards oxidation, and their behaviour under ionic bonding conditions.

When modelling the electronic structure of actinide materials, the most distinguishing feature is the increasing importance of correlations across the series from $U$ to $\mathrm{Cf}$, as the nature of the $f$-electrons changes from delocalized in the early actinides to localized in the later actinides. [6, 7] Electronic structure calculations, based on the local spin density approximation (LSDA), do not take into account strong on-site correlations beyond the homogeneous electron gas, and therefore can not adequately describe the localized phase of actinide materials. Thus the LSDA, 8] or even the generalized gradient approximation (GGA) [9] (which extends beyond the LSDA by taking into account charge density gradients), wrongly predicts a metallic ground state for $\mathrm{UO}_{2}, \mathrm{PuO}_{2}$ and $\mathrm{Pu}_{2} \mathrm{O}_{3}$, although the equilibrium lattice parameters 
and cohesive properties are found to be in rather good agreement with experiment. [9, 10, 11]

A number of schemes have been developed that augment the standard bandstructure framework to include the effects of strong correlations on the electronic structure. In the LDA+U approach [12] an effective Coulomb parameter $U$ is introduced that separates the $f$-manifold into the upper and lower Hubbard bands and removes $f$-degrees of freedom from the Fermi level. The more advanced dynamical mean-field theory (DMFT) approach provides a description of the competing trends towards localization on the one hand and itineracy on the other hand by taking into account the local quantum fluctuations missing in the static LDA+U treatment [13, 14]; albeit still at the cost of the introduction of the $U$ parameter. The hybrid density functional [15] theory implements an exchange-correlation functional where a fraction of the exact non-local exchange interaction from Hartree-Fock theory is mixed with the local or semi-local exchange energy of LSDA or GGA with the result that the troublesome effects of the known self-interaction error present in the standard LSDA and GGA calculations are reduced.

The self-interaction corrected (SIC)-LSD approach [16] used in the current work removes the self-interaction error that occurs in the LSDA, thereby leading to an improved description of the static Coulomb correlations of the f-electrons. The self-interaction correction associates an energy gain with electron localization, which competes with the opposing trend of band formation, providing a dual picture of combined localized and band like $f$-electrons. The method is fully $a b$-initio as both kinds of electrons are treated on an equal footing, with no adjustable parameters. A comparative study of $\mathrm{MnO}$, involving SIC-LSD, LDA+U, and the hybrid functional methods, was published by Kasinathan et al.. 17]

The present paper is organized as follows. In the following section, we give a short introduction to the SICLSD band structure method. In section III, we present our SIC-LSD results for the ground state properties of (A) the monoxides, (B) the sesquioxides, and (C) the dioxides where we also consider oxidation/reduction energies. In section IV, we give a summarizing discussion of the results, also concerning the relation between $f$ electron localization and oxidation by comparing to the lanthanide oxides. The conclusion of our paper is presented in section $\mathrm{V}$.

\section{THE SIC-LSD METHODOLOGY}

The LSD approximation to the exchange and correlation energy introduces an unphysical interaction of an electron with itself [16] which, although insignificant for extended band states, may lead to uncontrollable errors in the description of atomic-like localized states, for example the $f$-electrons in the later actinides. The SIC-LSD method [18, 19] corrects for this spurious self- interaction by adding to the LSD total energy functional an explicit energy contribution for an electron to localize. The resulting, orbital dependent, SIC-LSD total energy functional has the form

$$
E^{S I C-L S D}=E^{L S D}+E_{s o}-\Delta E_{s i c}
$$

where

$$
\begin{aligned}
E^{L S D} & =\sum_{\alpha}^{o c c .}\left\langle\psi_{\alpha}\left|-\nabla^{2}\right| \psi_{\alpha}\right\rangle+U[n]+V_{\text {ext }}[n] \\
& +E_{x c}^{L S D}\left[n_{\uparrow}, n_{\downarrow}\right] \\
E_{\text {so }} & =\sum_{\alpha}^{o c c .}\left\langle\psi_{\alpha}|\xi(\vec{r}) \vec{l} \cdot \vec{s}| \psi_{\alpha}\right\rangle \\
\Delta E_{\text {sic }} & =\sum_{\alpha}^{o c c .} \delta_{\alpha}^{S I C}=\sum_{\alpha}^{o c c .}\left\{U\left[n_{\alpha}\right]+E_{x c}^{L S D}\left[n_{\alpha}\right]\right\} .
\end{aligned}
$$

Here the sums run over all occupied electron states $\psi_{\alpha}$. As usual, the LSD total energy functional (2) is decomposed into the kinetic energy, the Hartree energy, the interaction energy with the atomic ions, and the exchange and correlation energy. The spin-orbit interaction (3) couples the band Hamiltonian for the spin-up and spindown channels, i.e. a double secular problem must be solved. The spin-orbit parameter,

$$
\xi(r)=-\frac{2}{c^{2}} \frac{d V}{d r},
$$

in atomic Rydberg units, is calculated from the selfconsistent potential. The self-interaction energy (4) consists of the self-Coulomb and self-exchange-correlation energies of the occupied orbitals $\psi_{\alpha}$ with the orbital charge density $n_{\alpha}$.

For itinerant states, the self-interaction $\delta_{\alpha}^{S I C}$ vanishes identically, while for localized (atomic-like) states $\delta_{\alpha}^{S I C}$ may be appreciable. Thus, the self-interaction correction constitutes a negative energy contribution gained by an electron upon localization, which competes with the band formation energy gained by the electron if allowed to delocalize and hybridize with the available conduction states. Different localized/delocalized configurations are realized by assuming different numbers of localized states - here $f$-states on actinide-atom sites. For $s$ - and $p$-states, $\delta_{\alpha}^{S I C}$ is never competitive compared to the corresponding gain in band formation energy, and turns out to be positive. Since the different localization scenarios constitute distinct local minima of the same energy functional, $E^{S I C-L S D}$, their total energies may be compared and the global energy minimum then defines the ground state total energy and the valence configuration of the actinide-ion. This latter is defined as the integer number of electrons available for band formation,

$$
N_{\text {val }}=Z-N_{\text {core }}-N_{S I C},
$$

where $Z$ is the atomic number, $N_{\text {core }}$ is the number of atomic core electrons, and $N_{S I C}$ is the number of SIClocalized $f$-electrons. In the remainder of this paper we 
will be using two interchangeable nomenclatures, $f^{n}$ and $\mathrm{A}^{m+}$, to describe the configuration of the actinide ion, implying $n=N_{S I C}$ and $m=N_{v a l}$, respectively. The total number of $f$-electrons may be larger than $n$, since, in addition to the $n$ localized $f$-states, the band states also contribute to the total $f$-count on a given ion. Note that our calculated valencies refer to the number of actinide electrons that contribute to bonding, and thus do not necessarily coincide with the nominal (ionic) valency of a compound: For $\mathrm{PuO}_{2}$ for example, the $\mathrm{Pu}^{4+}$ would agree with an ionic picture, while the $\mathrm{Pu}^{3+}$, and $\mathrm{Pu}^{5+}$ valency configurations would indicate more covalent behaviour.

The SIC-LSD approach is fully ab-initio, as both localized and delocalized states are expanded in the same set of basis functions and are, thus, treated on an equal footing. If no localized states are assumed, $E^{S I C-L S D}$ coincides with the conventional LSD functional, i.e., the Kohn-Sham minimum of the $E^{L S D}$ functional is also a local minimum of $E^{S I C-L S D}$.

Given the total energy functional $E^{S I C-L S D}$, the computational procedure is as for the LSD case, i.e. minimization is accomplished by iteration until selfconsistency. In the present work, the electron wavefunctions are expanded in the linear-muffin-tin-orbital (LMTO) basis functions. 20] The atomic spheres approximation (ASA) is used, whereby the crystal volume is divided into slightly overlapping atom-centered spheres of a total volume equal to the actual volume. A known shortcoming of the ASA is that different crystal structures have different degrees of overlap of the ASA spheres resulting in substantial relative errors in the evaluation of the total energy. While this inhibits the comparison of energies of different crystal structures, when comparing the energies of different localization scenarios within the same crystal structure, the ASA error is of minor influence. To improve the packing of the structure empty spheres have been introduced on high symmetry interstitial sites.

\section{RESULTS}

\section{A. Actinide Monoxides \\ 1. Background information}

There exists to date no convincing evidence that actinide oxides can form in the 1:1 stoichiometry. The experimental lattice parameters that we cite in Table 1 come from early reports on these compounds, and have so far not been confirmed. There have been no claims of bulk UO having ever been synthesized, and reports of an UO surface phase on $\mathrm{U}$ metal for low exposures to $\mathrm{O}, 21,22$ and UO thin films [23] could not be reproduced. 24] It has been suggested that the observed thin films actually represent uranium oxynitrides $\left(\mathrm{UN}_{x} \mathrm{O}_{1-x}\right)$ and oxycarbides $\left(\mathrm{UC}_{x} \mathrm{O}_{1-x}\right)$, that form in the presence of $\mathrm{N}_{2}$ or $\mathrm{C}$, and at low oxygen pressure. 25. It has similarly been con-
TABLE I: Actinide monoxide data. Column 2: Groundstate configuration. Column 3: Energy difference in $\mathrm{eV}$ between the ground state and the ideal ionic divalent configuration. Column 4: Groundstate density of states at the Fermi level (in units of states per $\mathrm{eV}$ and formula unit). Column 5: Calculated lattice parameters $a_{0}^{\text {calc }}$ (in $\AA$ ) . Column 6: Experimental lattice parameter $a_{0}^{\exp }$ (in $\AA$ ), where known (measurements on $\mathrm{UO}$, and $\mathrm{NpO}$ refer to thin film data, measurements on $\mathrm{PuO}, \mathrm{AmO}$, and $\mathrm{BkO}$ refer to bulk data).

\begin{tabular}{|c|c|c|c|c|c|}
\hline \hline $\mathrm{AO}$ & Config. & $\mathrm{E}_{G S^{-}}-\mathrm{E}_{I I}$ & $\mathrm{n}\left(E_{F}\right)$ & $\mathrm{a}_{0}^{\text {calc }}$ & $\mathrm{a}_{0}^{\text {exp }}$ \\
\hline $\mathrm{UO}$ & $\mathrm{f}^{1}\left(\mathrm{U}^{5+}\right)$ & -1.93 & 5.8 & 4.94 & $4.92^{a}$ \\
\hline $\mathrm{NpO}$ & $\mathrm{f}^{3}\left(\mathrm{~Np}^{4+}\right)$ & -1.73 & 1.1 & 4.99 & $5.01^{b}$ \\
\hline $\mathrm{PuO}$ & $\mathrm{f}^{5}\left(\mathrm{Pu}^{3+}\right)$ & -0.58 & 5.9 & 5.13 & $4.960^{c}$ \\
\hline $\mathrm{AmO}$ & $\mathrm{f}^{6}\left(\mathrm{Am}^{3+}\right)$ & -0.14 & 7.3 & 5.14 & $5.045^{c}$ \\
\hline $\mathrm{CmO}$ & $\mathrm{f}^{7}\left(\mathrm{Cm}^{3+}\right)$ & -1.14 & 2.6 & 5.01 & - \\
\hline $\mathrm{BkO}$ & $\mathrm{f}^{8}\left(\mathrm{Bk}^{3+}\right)$ & -0.65 & 1.4 & 4.97 & $4.964^{d}$ \\
\hline $\mathrm{CfO}$ & $\mathrm{f}^{9}\left(\mathrm{Cf}^{3+}\right)$ & -0.20 & 9.5 & 4.97 & - \\
\hline $\mathrm{EsO}$ & $\mathrm{f}^{10}\left(\mathrm{Es}^{3+}\right)$ & 0.00 & 4.0 & 4.92 & - \\
\hline $\mathrm{EsO}$ & $\mathrm{f}^{11}\left(\mathrm{Es}^{2+}\right)$ & 0.00 & 0.0 & 5.06 & - \\
\hline \hline
\end{tabular}

${ }^{a}$ Reference $23{ }^{b}$ Reference $30{ }^{c}$ Reference $28{ }^{d}$ Reference 29

cluded that neither bulk $\mathrm{NpO}$, nor a corresponding $\mathrm{NpO}$ surface phase will form. [26, 27] Preparation of $\mathrm{PuO}$ and AmO, 28] as well as possibly $\mathrm{BkO}$, 29] has been neither substantiated, nor has it been dismissed.

\section{SIC-LSD electronic structure}

We have calculated the electronic structure of the monoxides with the SIC-LSD method in order to establish the ground state properties for the hypothetical $\mathrm{NaCl}$ structure. Ferromagnetic arrangement of the spins has been assumed in these calculations. The results are summarized in Table I. We find the trivalent configuration to be energetically most favourable for all the monoxides, except $\mathrm{UO}$ and $\mathrm{NpO}$ that respectively prefer the $\mathrm{U}^{5+}$ and $\mathrm{Np}^{4+}$ ground state configurations. Concerning UO, it should be noted here that even though we find the $\mathrm{U}$ $f^{1}$ configuration to be the ground state, this energy scenario is close to degenerate with the fully itinerant LSD $\left(f^{0}\right)$ and $f^{2}$ scenarios, as shown in figure 11 This indicates that the $f$-electron manifold lingers between the localized and delocalized pictures, i.e. correlations are strong, but not to the degree for full localization to occur. This agrees with earlier results by Brooks et al., [31] where the electronic structure of UO was calculated assuming itinerant $f$-electrons, and which resulted in a calculated lattice parameter of $4.88 \AA$, i.e. only slighty overbinding with respect to the "experimental" value of $4.92 \AA$. The SIC-LSD calculated lattice parameters of the $f^{1}$ and $f^{2}$ configurations, respectively $4.94 \AA$ and $4.99 \AA$, indicate a slight overlocalization. Overall, the calculated lattice parameters are in rather good agreement with the early experimental values. However, as there is no convincing subsequent experimental evidence in support of the fact that monoxides really exist in nature, one should not put 


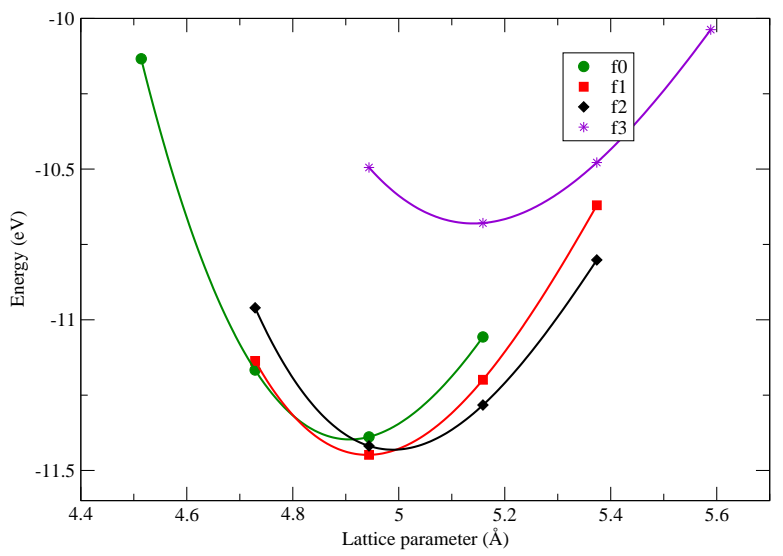

FIG. 1: Total energy of UO in the rocksalt structure assuming different localized/delocalized $f$-electron scenarios.

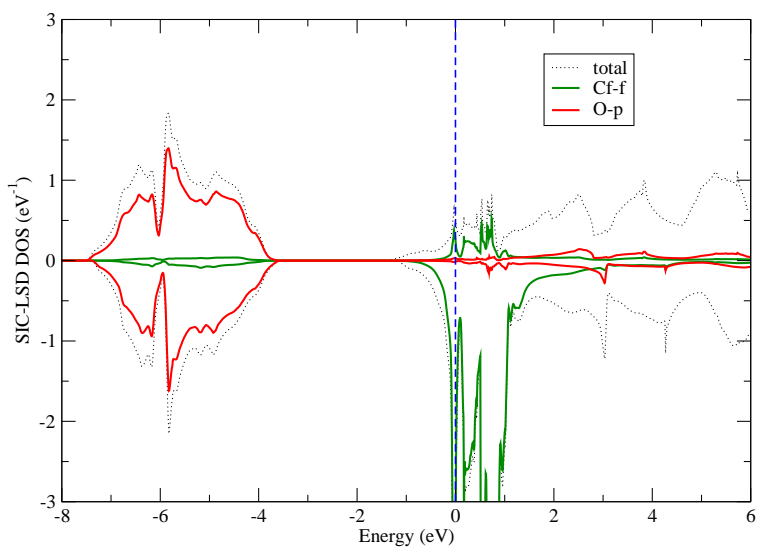

FIG. 2: Density of states for CfO in the ground state configuration with nine $f$-electrons localized on the $\mathrm{Cf}^{3+}$ ion. The majority (minority) spin components are displayed as positive (negative) values. The energy is measured in $\mathrm{eV}$ relative to the Fermi level, which falls amidst the minority $\mathrm{Cf} f$ bands. The localized Cf $f$ states are not shown.

too much weight on the agreement between theory and experiment.

For the monoxides beyond UO, the LSD configuration never becomes even remotely energetically favourable. For $\mathrm{NpO}$ a tetravalent ground state $\left(\mathrm{Np}^{4+} \equiv \mathrm{Np}\left(f^{3}\right)\right)$ is found, whereas a trivalent ground state configuration is established for the remaining monoxides. In their respective ground states, the monoxides are metallic, as can be seen from the non-zero density of states (DOS) at the Fermi energy (column 4 of Table \). As a representative example, the DOS of $\mathrm{CfO}$ is depicted in Fig. 2. The $\mathrm{O}$ atom has two unoccupied $p$-states, and in the corresponding monoxide, the $p$-band can accomodate two electrons from the actinide atom through charge transfer and hybridization, whilst the remaining valence electrons, including the delocalized $f$-electrons, start filling the conduction band, with the Fermi level pinned to the narrow $f$-band. The ionic insulating picture would be realized if additional $f$-states preferred to localize, i.e. in the divalent configuration. In Table (column 3) the calculated energy differences, $\mathrm{E}_{G S}-\mathrm{E}_{I I}$, between the ground state configuration and the divalent configuration are shown. It is clear that the nominal ionic $\mathrm{A}^{2+} \mathrm{O}^{2-}$ scenario does not become energetically favourable for any of the monoxides. Nevertheless this energy difference is seen to decrease from $\mathrm{UO}$ to $\mathrm{AmO}$, and again from $\mathrm{CmO}$ to $\mathrm{CfO}$, which is in agreement with the trends towards increasing localization due to the actinide contraction. At $\mathrm{AmO}$ the trivalent configuration is thus only marginally more favourable than the divalent half-filled shell configuration, and at EsO degeneracy occurs between the two configurations. The overall non-ionic ground state picture for the monoxides that emerges from our SICLSD calculations confirms the results from early molecular cluster calculations on the heavy actinide monoxides by Gubanov et al., 32] where considerable covalency due to mixing of $\mathrm{O}-p$ and A- $f$ orbitals was found.

\section{B. Actinide Sesquioxides}

\section{Background information}

Bulk phases of $\mathrm{U}_{2} \mathrm{O}_{3}$ as well as $\mathrm{Np}_{2} \mathrm{O}_{3}$ do not exist in nature, and have never been synthesized, but thin films of $\mathrm{Np}_{2} \mathrm{O}_{3}$ have been found to form on the surface of $\mathrm{Np}$ metal.27 From $\mathrm{Pu}$ onwards, the sesquioxides are stable, and have been synthesized. Beyond $\mathrm{Pu}$, the sesquioxides crystallize in three different crystal structures, respectively the hexagonal $\mathrm{La}_{2} \mathrm{O}_{3}$ structure (A-form), the cubic $\mathrm{Mn}_{2} \mathrm{O}_{3}$ structure (C-form), and the monoclinic $\mathrm{Sm}_{2} \mathrm{O}_{3}$ structure (B-form). $\mathrm{Pu}_{2} \mathrm{O}_{3}$ has been synthesized only in the $\mathrm{A}$ - and $\mathrm{C}$-forms. The XPS measurements on sesquioxides from $\mathrm{Pu}_{2} \mathrm{O}_{3}$ to $\mathrm{Cf}_{2} \mathrm{O}_{3}$ have been reported, 33, 34, 35] and the absence of features at the Fermi level points towards the localized nature of the $5 f$ electrons in these compounds. This indicates that they are semiconductors or insulators, in agreement with the ideal nominal picture of $\mathrm{A}_{2}^{3+} \mathrm{O}_{3}^{2-}$, although no values for the energy gaps can be found in the literature.

There exist relatively few calculations of the electronic structure of the actinide sesquioxides. Prodan et al. 36] have studied $\mathrm{Pu}_{2} \mathrm{O}_{3}$ (A-form) with the help of hybrid density functional theory, comparing a number of functionals. They have found that unlike in the LSD and GGA approximations, using the Heyd-Scuseria- 
TABLE II: Actinide sesquioxide data: Column 2: Ground state configuration of actinide-ion. Column 3: Calculated energy gap, $\mathrm{E}_{\text {gap }}$, (in eV). Columns 4 and 5: Calculated, $\mathrm{V}_{\text {calc }}$, and experimental 30], $\mathrm{V}_{\text {exp }}$, equilibrium volume(s) in in units of $\AA^{3}$ per formula unit.

\begin{tabular}{|c|r|r|r|r|}
\hline \hline Compound & Ground state & $\mathrm{E}_{\text {gap }}$ & $\mathrm{V}_{\text {calc }}$ & $\mathrm{V}_{\text {exp }}$ \\
\hline \multicolumn{5}{|c|}{ C-type sesquioxides } \\
\hline \hline $\mathrm{U}_{2} \mathrm{O}_{3}$ & $\mathrm{f}^{2}\left(\mathrm{U}^{4+}\right)$ & 0.00 & 83.17 & - \\
\hline $\mathrm{Np}_{2} \mathrm{O}_{3}$ & $\mathrm{f}^{3}\left(\mathrm{~Np}^{4+}\right)$ & 0.00 & 84.40 & - \\
\hline $\mathrm{Pu}_{2} \mathrm{O}_{3}$ & $\mathrm{f}^{5}\left(\mathrm{Pu}^{3+}\right)$ & 0.75 & 89.42 & 82.73 \\
\hline $\mathrm{Am}_{2} \mathrm{O}_{3}$ & $\mathrm{f}^{6}\left(\mathrm{Am}^{3+}\right)$ & 0.44 & 88.54 & 83.64 \\
\hline $\mathrm{Cm}_{2} \mathrm{O}_{3}$ & $\mathrm{f}^{7}\left(\mathrm{Cm}^{3+}\right)$ & 0.32 & 86.98 & 83.10 \\
\hline $\mathrm{Bk}_{2} \mathrm{O}_{3}$ & $\mathrm{f}^{8}\left(\mathrm{Bk}^{3+}\right)$ & 0.38 & 83.41 & 80.63 \\
\hline $\mathrm{Cf}_{2} \mathrm{O}_{3}$ & $\mathrm{f}^{9}\left(\mathrm{Cf}^{3+}\right)$ & 0.47 & 82.60 & 79.59 \\
\hline \multicolumn{5}{|c|}{$\mathrm{A}-$ type sesquioxides } \\
\hline \hline $\mathrm{Pu}_{2} \mathrm{O}_{3}$ & $\mathrm{f}^{5}\left(\mathrm{Pu}^{3+}\right)$ & 2.43 & 74.06 & 75.49 \\
\hline $\mathrm{Am}_{2} \mathrm{O}_{3}$ & $\mathrm{f}^{6}\left(\mathrm{Am}^{3+}\right)$ & 2.54 & 73.34 & 74.73 \\
\hline $\mathrm{Cm}_{2} \mathrm{O}_{3}$ & $\mathrm{f}^{7}\left(\mathrm{Cm}^{3+}\right)$ & 3.07 & 72.40 & 74.53 \\
\hline $\mathrm{Bk}_{2} \mathrm{O}_{3}$ & $\mathrm{f}^{8}\left(\mathrm{Bk}^{3+}\right)$ & 2.73 & 70.10 & 72.71 \\
\hline $\mathrm{Cf}_{2} \mathrm{O}_{3}$ & $\mathrm{f}^{9}\left(\mathrm{Cf}^{3+}\right)$ & 1.78 & 69.33 & 71.43 \\
\hline \hline
\end{tabular}

Ernzerhof (HSE) screened Coulomb functional leads to an insulating anti-ferromagnetic solution, in good agreement with experiment, with the calculated gap value of $2.78 \mathrm{eV}$ (3.50 eV for the Perdew-Burke-Ernzerhof (PBE0) hybrid functional). The antiferromagnetic insulating nature of $\mathrm{Pu}_{2} \mathrm{O}_{3}$ has similarly been retrieved from electronic structure calculations by respectively Jomard et al.[11] and Sun et al. [37], based on the (LDA/GGA)+U approximation. Their gap values are found to depend strongly on the value chosen for the electron-electron interaction $\mathrm{U}$. For $\mathrm{U}=4 \mathrm{eV}$, and depending on the details of the functional, energy gaps ranging from 1 to $2 \mathrm{eV}$ were obtained.

\section{SIC-LSD electronic structure}

In the present work the electronic structures of the sesquioxides from $\mathrm{U}_{2} \mathrm{O}_{3}$ to $\mathrm{Cf}_{2} \mathrm{O}_{3}$ has been calculated for both the cubic $\mathrm{C}$-form and the hexagonal $\mathrm{A}$-form. The results are summarized in Table \, The hexagonal Atype structure has space group P $\overline{3} \mathrm{~m} 1$ (no. 164). The unit cell contains one formula unit with one Oxygen at the origin, two Oxygens at $\pm\left(1 / 3,2 / 3, \mathrm{z}_{O}\right)$ and two actinide atoms at $\pm\left(1 / 3,2 / 3, \mathrm{z}_{A}\right)$. While the $\mathrm{c} /$ a ratio has been measured for all the actindes from $\mathrm{Pu}$ to $\mathrm{Cf}$, the internal parameters are only known for $\mathrm{Pu}\left(\mathrm{z}_{O}=0.6451\right.$ and $\left.\mathrm{z}_{P u}=0.2408\right)$. [30] Hence we have performed our calculations for this structure, with the experimental c/a ratios but using the internal parameters of $\mathrm{Pu}_{2} \mathrm{O}_{3}$ for all the other actinide sesquioxides. [38] The C-type structure, also known as bixbyite, has been approximated by the fluorite $\mathrm{AO}_{2}$ structure, with $1 / 4$ of the $\mathrm{O}$ atoms removed, i.e. from four formula units in a conventional simple cubic supercell the oxygen atoms at the origin and the cube center were replaced by empty spheres. No relaxation of

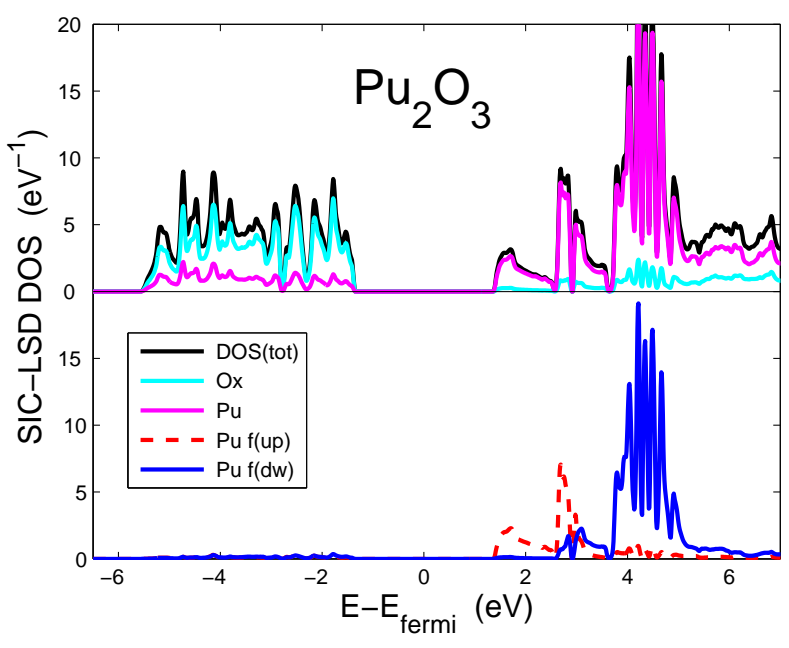

FIG. 3: Density of states for A-type $\mathrm{Pu}_{2} \mathrm{O}_{3}$ in the trivalent ground state configuration. The energy is in $\mathrm{eV}$ relative to the Fermi level, which is situated midgap. The DOS is in units of $\mathrm{eV}^{-1}$ per formula unit. Upper panel: Total (black), $\mathrm{Pu}$ (violet), and $\mathrm{O}$ (light blue) DOS's. Lower panel: The corresponding $\mathrm{Pu}-f$ majority (red), and minority (blue) spin decomposed DOS's. Only the itinerant $f$-states are shown.

the atomic coordinates has been attempted. The trivalent actinide configuration is found to be energetically favourable for all the compounds, apart from $\mathrm{U}_{2} \mathrm{O}_{3}$ and $\mathrm{Np}_{2} \mathrm{O}_{3}$, for which the tetravalent ground state configuration is preferred. Incidentally, these are also the only sesquioxides that do not occur in nature. In the trivalent ground state, the sesquioxides are found to be insulators, with energy gaps of around $0.5 \mathrm{eV}$ for the $\mathrm{C}$-form, and around $2.7 \mathrm{eV}$ for the A-form. The corresponding DOS for the A-type $\mathrm{Pu}_{2} \mathrm{O}_{3}$ is shown in Fig. 3

Only the ferromagnetic ordering was investigated for the cubic structure, whilst only the antiferromagnetic ordering of spins was studied for the hexagonal structures, and the values for the insulating gaps in Table $\llbracket$ refer to these respective magnetic orderings. The bulk moduli for A-type $\mathrm{Pu}_{2} \mathrm{O}_{3}, \mathrm{Am}_{2} \mathrm{O}_{3}, \mathrm{Cm}_{2} \mathrm{O}_{3}, \mathrm{Bk}_{2} \mathrm{O}_{3}$, and $\mathrm{Cf}_{2} \mathrm{O}_{3}$ are similar in magnitude, calculated to be respectively, 158 , $158,168,166$, and $174 \mathrm{GPa}$. No experimental measurements of the bulk moduli seem to exist, but the value for $\mathrm{Pu}_{2} \mathrm{O}_{3}$ lies within the range of values obtained by Prodan et al. [36] and Jomard et al. 11] for a suite of different functionals (from $110 \mathrm{GPa}$ to $181 \mathrm{GPa}$ ). As can be seen from Table III the calculated equilibrium volumes of the A-type sesquioxides are found to be in good agreement with experiment. This agreement is somewhat less satisfying for the C-type sesquioxides, where the volume is overstimated by up to $4 \%$, which might be related to our approximate treatment of the actual bixbyite structure. 


\section{Actinide Dioxides}

\section{Background information}

The actinide dioxides from $\mathrm{U}$ to $\mathrm{Cf}$ have all been synthesized, crystallizing in the fluorite $\left(\mathrm{CaF}_{2}\right)$ structure. They are also the most relevant systems for applications and, as a consequence, are the most studied actinide oxide compounds, both experimentally and theoretically. 9, 11, 33, 35, 37, 39, 40, 41, 42, 43, 44, 45, 46, 47, 48, 49, 50] On the experimental side, a comprehensive summary of their electronic, magnetic, transport and optical properties can be found in Troć et al..48] The absence of features at the Fermi level in the observed XPS spectra 35. indicates that all the dioxides are semiconductors or insulators. However, specific information regarding the gap is only known for $\mathrm{UO}_{2}, \mathrm{NpO}_{2}$, and $\mathrm{PuO}_{2}$. A combined XPS and BIS spectrum for $\mathrm{UO}_{2}$ finds an $\mathrm{O}(2 \mathrm{p}) \rightarrow \mathrm{U}(6 \mathrm{~d})$ gap of about $5 \mathrm{eV}$.49] This value for the energy gap is similar to the one observed for $\mathrm{ThO}_{2}$, but with the difference that in $\mathrm{UO}_{2}$ two occupied rather well localized $f$-states are situated in the gap, with a $5 f^{2} \rightarrow 5 f^{1} 6 s d$ transition energy of $2-3 \mathrm{eV}$. [49, 51] As the nuclear charge increases from $\mathrm{Th}$ to $\mathrm{Pu}$, the $f$-states move to lower energies. In $\mathrm{ThO}_{2}$ the empty $f$-states are situated in the Th-sd derived conduction band. In $\mathrm{UO}_{2}$ and $\mathrm{NpO}_{2}$ the $f$-states are occupied and situated in the energy band gap, whereas in $\mathrm{PuO}_{2}$ they are situated at the top of the $\mathrm{O}-p$ derived valence band. 33. The measured activation energies, $\mathrm{E}_{a}=0.2 \mathrm{eV}$ in $\mathrm{UO}_{2}, \mathrm{E}_{a}=0.4 \mathrm{eV}$ in $\mathrm{NpO}_{2}$, and $\mathrm{E}_{a}=1.8 \mathrm{eV}$ in $\mathrm{PuO}_{2}$, confirm this trend. 50]

Electronic structure calculations, in particular for $\mathrm{UO}_{2}$ and $\mathrm{PuO}_{2}$, have shown that the itinerant $f$-electron picture is not adquate for describing these compounds. It has emerged that the LSD approximation wrongly predicts metallic behaviour, [9, 11, 39] demonstrating the need to go beyond the homogeneous electron gas in describing the strong on-site $f$-electron correlations. So far, a number of calculations have shown that an insulating solution can be obtained when using improved descriptions of electron correlations such as those contained in the LDA(GGA)+U[11, 37, 40, 41], DMFT 42], SICLSD [43] and hybrid functional methodologies. [44]

\section{SIC-LSD electronic structure}

In the present paper the electronic structures of the dioxides from $\mathrm{UO}_{2}$ to $\mathrm{CfO}_{2}$ has been calculated using the SIC-LSD method. As can be seen from Table III, a tetravalent ground state configuration is found for all the dioxides, except $\mathrm{UO}_{2}$ where the pentavalent $\left(\mathrm{U}\left(f^{1}\right)\right)$ configuration is energetically more favourable. Actually, the pentavalent $\mathrm{U}\left(f^{1}\right)$ and tetravalent $\mathrm{U}\left(f^{2}\right)$ configurations are energetically close, with the $f^{1}$ configuration being more favourable by some $100 \mathrm{meV}$. Our calculations refer to $\mathrm{T}=0 \mathrm{~K}$, whereas the experimental evidence, which clearly indicates a tetravalent (insulating) $\mathrm{UO}_{2}$, mostly refers to room temperature conditions. Experiments also indicate a lattice expansion with temperature, 52, 53. but the $f^{1}$ to $f^{2}$ localization transition that our calculations seem to predict has not been observed experimentally. The prediction of an $\mathrm{U}-f^{1}$ goundstate configuration could possibly be related to the tetrad effect (multiplet formation energy), [54, 55, 56] which is ignored in our calculation, and which favours the $f^{2}$ configuration over the $f^{1}$ configuration. In the following, when comparing to experiment, we will be referring to the tetravalent $\mathrm{UO}_{2}$ configuration as the ground state configuration.

All the dioxides (including the tetravalent $\mathrm{UO}_{2}$ ) are predicted to be insulators. The AFII magnetic ordering (i.e. ferromagnetically ordered planes stacked antiferrormagnetically along the [111] crystal direction) has been assumed in the dioxide calculations to which the SIC-LSD data in Table III refer. With respect to the band gap of $\mathrm{UO}_{2}$, a value of $\mathrm{E}_{g a p}=2.6 \mathrm{eV}$ is found, which is smaller than the experimentally observed $p \rightarrow d$ gap, and which should not be compared to the optical gap referred to in the $\mathrm{GGA}+\mathrm{U}(1.8 \mathrm{eV})$, and $\mathrm{HSE}(2.4 \mathrm{eV})$ calculations. The SIC-LSD, being a one-electron ground state theory, does not give accurate removal energies of localized states, on account of the missing screening and relaxation effects, predicting them at too high binding energies, in disagreement with spectroscopy. As a result, the SIC-LSD calulations do not reproduce the correct position of the occupied $f$-peak which is expected to be situated in the gap for both $\mathrm{UO}_{2}$ or $\mathrm{NpO}_{2}$. The Mott-insulating character $(f \rightarrow f$ transition) of the early dioxides [40, 44] is thus not reproduced in our calculations, which instead describe the entire series as charge transfer insulators $\left(p \rightarrow f\right.$ transition). From $\mathrm{PuO}_{2}$ onwards, the occupied $f$-states are situated at or below the valence band maximum, and the charge transfer picture becomes adequate for describing the nature of the gap. There exist only a few calculations dealing with the electronic structure of the heavy actinide dioxides. 32, 44, 45. In the following we make a detailed comparison with the recent results obtained by Prodan et al. 44] using the hybrid functional theory.

Both the SIC-LSD and the hybrid functional calculations determine the antiferromagnetic ground state configuration as energetically most favourable. As can be seen in Table III our calculated energy gaps are smaller than the corresponding HSE gaps, but the trends agree, i.e. the gaps decrease from $\mathrm{PuO}_{2}$ to $\mathrm{CmO}_{2}$, and then again from $\mathrm{BkO}_{2}$ onwards. This reflects the gradual progression towards lower energy of the unoccupied $f$-states, with increasing atomic number. The calculated lattice parameters are in very good agreement with experiment, within $1 \%$, as seen in both Table III and figure 4. We should note here that the experimentally observed lattice parameters refer to room temperature measurements, 30. and are thus on average larger than the values one would expect for $\mathrm{T}=0 \mathrm{~K}$ by approximately $0.011 \AA$. [59] The consistent overestimation of the lattice parameters is quite common for SIC-LSD, which has tendency to slightly 
TABLE III: Actinide dioxide data: Column 2: ground state configuration. Columns 3 and 4: Energy gaps, E $\mathrm{E}_{\text {gap }}^{\text {SIC }}$ (this work) and $\mathrm{E}_{\text {gap }}^{H S E}$ (reference 44), respectively (in units of eV). Columns 5 and 6 : calculated, $\mathrm{a}_{0}^{S I C}$, and experimental $\mathrm{a}_{0}^{\exp }$ lattice parameters (in $\AA$ ). Column 7 and 8: calculated, $\mathrm{B}_{0}^{S I C}$, and experimental, $\mathrm{B}_{0}^{S I C}$, bulk modulii (in units of GPa).

\begin{tabular}{|c|c|c|c|c|c|c|c|}
\hline \hline & Config. & $\mathrm{E}_{\text {gap }}^{\text {SIC }}$ & $\mathrm{E}_{\text {gap }}^{\text {HSE }}$ & $\mathrm{a}_{0}^{\text {SIC }}$ & $\mathrm{a}_{0}^{\text {exp }}$ & $\mathrm{B}_{0}^{\text {SC }}$ & $\mathrm{B}_{0}^{\text {exp }}$ \\
\hline $\mathrm{UO}_{2}$ & $\mathrm{f}^{1}\left(\mathrm{U}^{5+}\right)$ & 0 & 2.4 & 5.40 & 5.470 & 219 & $207^{b}$ \\
\hline $\mathrm{UO}_{2}$ & $\mathrm{f}^{2}\left(\mathrm{U}^{4+}\right)$ & 2.6 & 2.4 & 5.47 & 5.470 & 219 & $207^{b}$ \\
\hline $\mathrm{NpO}_{2}$ & $\mathrm{f}^{3}\left(\mathrm{~Np}^{4+}\right)$ & 2.3 & 3.1 & 5.46 & 5.433 & 217 & $200^{b}$ \\
\hline $\mathrm{PuO}_{2}$ & $\mathrm{f}^{4}\left(\mathrm{Pu}^{4+}\right)$ & 1.2 & 2.7 & 5.44 & 5.396 & 214 & $178^{b}$ \\
\hline $\mathrm{AmO}_{2}$ & $\mathrm{f}^{5}\left(\mathrm{Am}^{4+}\right)$ & 0.8 & 1.6 & 5.42 & 5.374 & 209 & $205^{b}$ \\
\hline $\mathrm{CmO}_{2}$ & $\mathrm{f}^{6}\left(\mathrm{Cm}^{4+}\right)$ & 0.4 & 0.4 & 5.37 & 5.359 & 212 & $218^{c}$ \\
\hline $\mathrm{BkO}_{2}$ & $\mathrm{f}^{7}\left(\mathrm{Bk}^{4+}\right)$ & 1.0 & 2.5 & 5.36 & 5.331 & 221 & - \\
\hline $\mathrm{CfO}_{2}$ & $\mathrm{f}^{8}\left(\mathrm{Cf}^{4+}\right)$ & 0.6 & 2.0 & 5.36 & 5.310 & 210 & - \\
\hline \hline
\end{tabular}

${ }^{a}$ Reference $30{ }^{b}$ Reference $57^{c}$ Reference 58

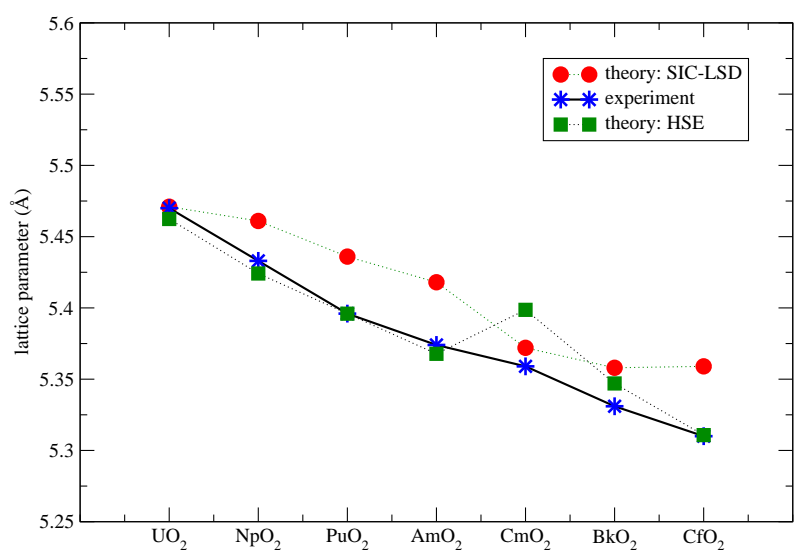

FIG. 4: Lattice parameters of the actinide dioxides. Experimental values [30] (blue stars) are compared to the theoretical SIC-LSD values (red circles), and the theoretical HSE values (green squares) read from the corresponding figure 1 in reference 44 .

overlocalize.

The overall experimental situation with regard to $\mathrm{CmO}_{2}$ is still not fully understood. 60] It has been shown that, for a range of off-stoichiometric compounds $\mathrm{CmO}_{2-x}$, both the lattice parameter and magnetic moment increase with $x$. 61, 62, 63] Given the expected moments of $\mathrm{Cm}^{3+}\left(5 f^{7} ; \mu_{e f f}=7.94 \mu_{B}\right)$ and $\mathrm{Cm}^{4+}\left(5 f^{6}\right.$; $\left.\mu_{e f f}=0 \mu_{B}\right)$ it was noted that this trend indicates an increase in the number of $\mathrm{Cm}^{3+}$ impurities as we move away from stoichiometry. Based on the ionic picture, one accordingly expects the $\mathrm{Cm}^{4+}$ ground state configuration for the stoichiometric compound, which however seems to be contradicted by the fact that in samples very close to stoichimetry, 64] susceptibility measurements give the effective moment as high as $\mu_{e f f}=3.36 \mu_{B}$. Attempts to explain this discrepancy, range from a possible existence of an impurity phase, not registered in the experiments, to assuming a covalent, rather than an ionic, picture for the ground state. 60. It has also been conjectured that a more complex ground state which includes some admixture of the excited $J=1$ state of $\mathrm{Cm}^{4+}$ might be required. 64.

The lattice parameter measurements yield two different values for $\mathrm{CmO}_{2}$, depending on whether the shortlived curium isotope of mass number 244 is used ( $a=$ $5.372 \AA), 65]$ or the long-lived curium isotope of mass number 248 is used $(a=5.359 \AA)$. 62 The reason for this discrepancy has been traced to the self-radiation induced expansion that occurs with the considerably more active ${ }^{244} \mathrm{Cm}$ isotope. 62, 63 It is the ${ }^{248} \mathrm{CmO}_{2}$ lattice parameter, i.e. without the effect of self-heating, that should be of relevance when comparing to the calculated lattice parameters.

From the SIC-LSD calculations we find the ground state of $\mathrm{CmO}_{2}$ to be tetravalent, and the corresponding lattice parameter is in good agreement with experiment. In figure 5 we show the DOS of $\mathrm{CmO}_{2}$ in the $\mathrm{Cm}^{4+}\left(f^{6}\right)$ ground state configuration. The compound is found to be insulating, with the Fermi level positioned between the completely filled $\mathrm{O}-p$ band, and the one remaining delocalized empty majority $f$-spin state that strongly hybridizes with the O- $p$ states. The resulting electronic structure is thus quite different from the hybrid functional picture, where the HSE applied by Prodan et al. results in metallic $\mathrm{CmO}_{2}$. The authors suggest a covalent picture with significant $\mathrm{Cm}^{3+}$ character, as a result of the $\mathrm{Cm}$ trying to achieve the stable half-filled $f$-shell configuration. Consequently some of the $\mathrm{O} p$-states are charge transferred to the $\mathrm{Cm}$-ion, resulting in the Fermi level cutting accross the top of the hybridized $\mathrm{O} p-\mathrm{Cm}$ $f$ band. However, unlike for all the other actinide dioxides, the lattice parameter of $\mathrm{CmO}_{2}$, calculated using the HSE functional, deviates considerably from the measured value, as can be seen from Fig. 4 .

With respect to the SIC-LSD calculations, one could envisage that a $\mathrm{Cm}^{3+}$ configuration in $\mathrm{CmO}_{2}$ could be energetically favourable, given the associated half-filled $f$-shell. Due to one less $f$-state taking part in bonding, as compared to the $\mathrm{Cm}^{4+}$, this in return could explain the slight increase in the experimentally observed lattice parameter of $\mathrm{CmO}_{2}$. As it turns out, however, the total energy of the trivalent configuration is higher by $0.68 \mathrm{eV}$, indicating that the $\mathrm{Cm}^{4+}$ configuration is energetically very stable. The reason for this stability is related to the fact that localizing an additional electron in $\mathrm{CmO}_{2}$ results in the Fermi level moving down into the $p$-band. This implies a depopulation of the $p$-band, as charge is transferred to the low lying $f$-levels, and the associated loss in respectively the Madelung and hybridization energies is significantly larger than the gain in the $f$-localization energy. This charge transfer picture is similar to the picture found in the hybrid functional theory. However, in variance to the conclusion of that work, we find the corresponding trivalent configuration to be energetically unfavourable.

Although our calculated ground state of $\mathrm{CmO}_{2}$ finds a 


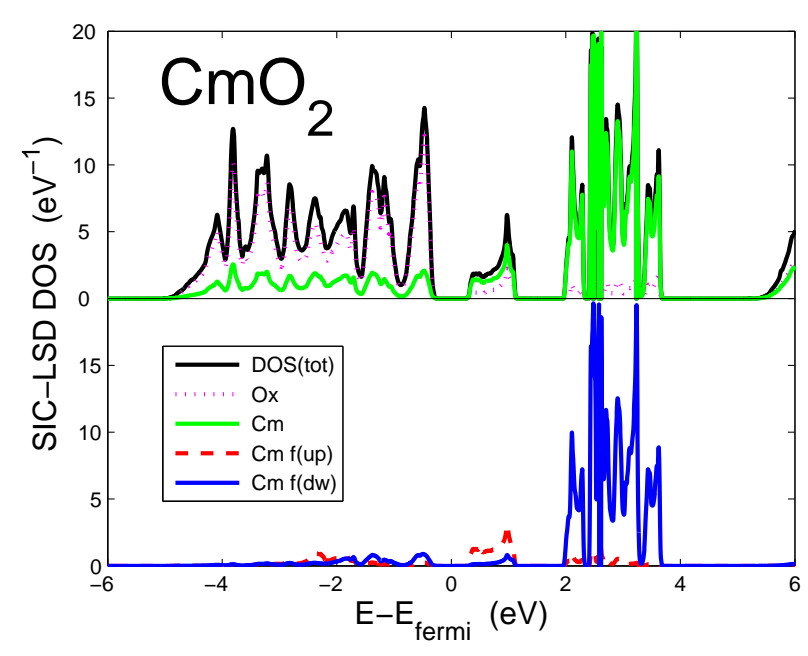

FIG. 5: DOS of $\mathrm{CmO}_{2}$. Upper panel: Total (black), Cm (green), and O (red dotted) DOS's. Lower panel: The corresponding Cm- $f$ majority (red), and minority (blue) spin decomposed DOS's. Only the itinerant $f$-states are shown.

localized $f^{6} \mathrm{Cm}$ ion, the system turns out to be magnetic. The ideal $f^{6}$ ion has $J=0$ and hence also $\left.<S_{z}\right\rangle=0$ and $\left\langle L_{z}\right\rangle=0$, but this state is a linear combination of several 6-electron Slater determinants, which we cannot treat in our SIC-LSD scheme. Rather, it may be represented by a single Slater determinant having $\left\langle S_{z}\right\rangle=3$ and $\left\langle L_{z}\right\rangle=-3$ (simulating antiparallel $S=3$ and $L=3$ ), which constitutes the starting point for our calculations before iteration to selfconsistency. Spin-orbit coupling and hybridization significantly distort this initial configuration, in fact almost quenching the orbital moment. The total spin and orbital moment projections along the $z$-axis are calculated to be $\left\langle S_{z}\right\rangle=2.73$ and $\left\langle L_{z}\right\rangle=-0.25$ (in units of $\hbar)$. This comes from contributions from the localized $f^{6}$ ion $\left(<S_{z}\right\rangle=2.76$ and $\left.\left\langle L_{z}\right\rangle=-1.15\right)$ and from the delocalized f-band states $\left(\left\langle S_{z}\right\rangle=-0.03\right.$ and $\left.\left.<L_{z}\right\rangle=+0.93\right)$. The latter are largely tails of the $\mathrm{O}$ p-band states, which inside the atomic sphere around $\mathrm{Cm}$ attain $f$-character. The total number of delocalized $f$-character states is 0.86 , leading to a total $f$-count of 6.86 , which reflects a significant $f$-hybridization with the $\mathrm{O}$ p bands. Assuming now, that the magnetic moment is given as $\mu=<2 S_{z}+L_{z}>\mu_{B}$, we arrive at a total moment of $\mu=5.21 \mu_{B}$ per $\mathrm{Cm}$ atom, which exceeds the experimental moment of $\mu_{\text {eff }}=3.36 \mu_{B}$, 64 however demonstrating that the insulating state of $\mathrm{CmO}_{2}$ may be magnetic. The quoted experimental moment is extracted from the large temperature magnetic susceptibility, while the present theory is valid only at $T=0$. Furthermore, as stated, the fact that quantum fluctuations (more than one Slater determinant representing the localized $f^{6}$ shell), are not possible within the SIC-LSD approach, might be a serious limitation with respect to a proper description of magnetic properties. Thermal fluctuations can be considered using the so-called local SIC (LSIC) approach, implemented in the multiple scattering theory, in combination with the coherent potential approximation (CPA) and disordered local moment (DLM) theory. 66, 67]

\section{Oxidation and Reduction Energies}

Uranium metal readily oxidizes to form a range of super-stoichiometric oxide compounds, $\mathrm{UO}_{2+x}$, as well as stoichiometric compounds, $\mathrm{UO}_{2}, \mathrm{U}_{3} \mathrm{O}_{8}, \mathrm{UO}_{3}$, and $\mathrm{U}_{2} \mathrm{O}_{5}$. Sub-stoichiometric $\mathrm{UO}_{2-x}$ exists, but $\mathrm{U}_{2} \mathrm{O}_{3}$ for example is not found in the literature. In this section we will investigate oxidation/reduction of the actinide dioxides, based on the SIC-LSD total energies involved in the different delocalization/localization transitions of the $f$-electrons. To model the oxidation process

$$
\mathrm{AO}_{2}+\frac{1}{8} \mathrm{O}_{2} \rightarrow \mathrm{AO}_{2.25}
$$

one additional $\mathrm{O}$ is introduced into a four formula units $\mathrm{AO}_{2}$ supercell, i.e. we define the oxidation energy

$$
E^{o x}=\frac{1}{4}\left[E\left(\mathrm{~A}_{4} \mathrm{O}_{9}\right)-E\left(\mathrm{~A}_{4} \mathrm{O}_{8}\right)-\frac{1}{2} E\left(\mathrm{O}_{2}\right)\right] .
$$

The $\mathrm{CaF}_{2}$ structure is assummed to remain undistorted, i.e. relaxation effects are not taken into account. For the $\mathrm{AO}_{2}$ reduction process we consider the reaction

$$
\mathrm{AO}_{2} \rightarrow \mathrm{AO}_{1.5}+\frac{1}{4} \mathrm{O}_{2}
$$

In the supercell used for modelling the reaction, two $\mathrm{O}$ atoms are removed from the four formula units $\mathrm{AO}_{2}$ supercell. Correspondingly, the reduction energy is

$$
E^{r e d}=\frac{1}{4}\left[E\left(\mathrm{~A}_{4} \mathrm{O}_{6}\right)+E\left(\mathrm{O}_{2}\right)-E\left(\mathrm{~A}_{4} \mathrm{O}_{8}\right)\right] .
$$

The compound $\mathrm{A}_{4} \mathrm{O}_{6}$ is basically a two formula unit supercell of the sesquioxide $\mathrm{A}_{2} \mathrm{O}_{3}$ in the previously described approximation to the bixbyite structure.

The supercell total energies $\mathrm{E}\left(\mathrm{A}_{4} \mathrm{O}_{8}\right)$ and $\mathrm{E}\left(\mathrm{A}_{4} \mathrm{O}_{6}\right)$ have been evaluated at the ground state valency configurations established in the preceding sections for respectively $\mathrm{AO}_{2}$ and $\mathrm{A}_{2} \mathrm{O}_{3}$. For $\mathrm{A}_{4} \mathrm{O}_{9}$, the SICLSD calculations find the following ground state configurations: $\mathrm{U}_{4}^{5+} \mathrm{O}_{9}, \mathrm{~Np}_{4}^{5+} \mathrm{O}_{9}, \mathrm{Pu}_{2}^{4+} \mathrm{Pu}_{2}^{5+} \mathrm{O}_{9}, \mathrm{Am}_{4}^{4+} \mathrm{O}_{9}$, $\mathrm{Cm}_{3}^{4+} \mathrm{Cm}^{5+} \mathrm{O}_{9} / \mathrm{Cm}_{4}^{4+} \mathrm{O}_{9}, \mathrm{Bk}_{4}^{4+} \mathrm{O}_{9}$, and $\mathrm{Cf}_{4}^{4+} \mathrm{O}_{9}$. The corresponding total energy minima $\mathrm{E}\left(\mathrm{A}_{4} \mathrm{O}_{9}\right)$ are used in the oxidation energy calculations of Eq.(17). Compared to the tetravalent dioxide, $\mathrm{A}_{4} \mathrm{O}_{8}$, we observe that in $\mathrm{A}_{4} \mathrm{O}_{9}$, the valency trend is from pentavalent in the early actinides to tetravalent in the late actinides, indicating that the inclusion of $\mathrm{O}$ impurities into the dioxide leads to $f$ electron delocalization in the early actinide oxides $\mathrm{U}_{4} \mathrm{O}_{9}$, $\mathrm{Np}_{4} \mathrm{O}_{9}$, and $\mathrm{Pu}_{4} \mathrm{O}_{9}$, whereas from $\mathrm{Am}_{4} \mathrm{O}_{9}$ onwards the compounds remain tetravalent. On the other hand the 


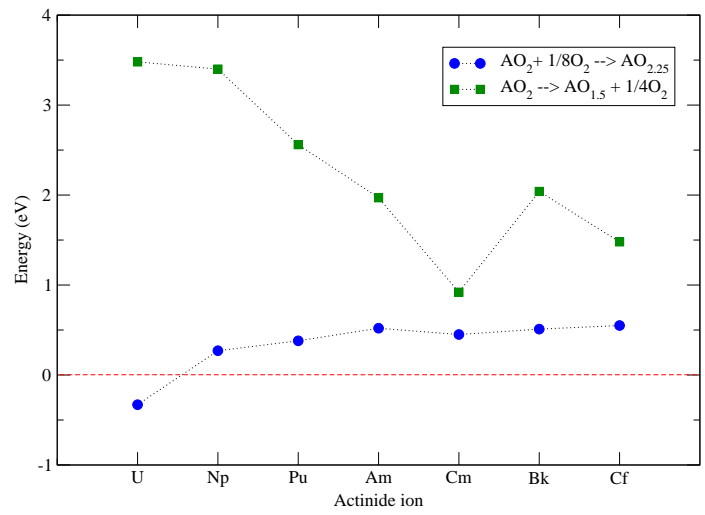

FIG. 6: Oxidation (circles) and reduction (squares) energies of the actinide dioxides according to the definitions in Eqs. (7) and (9). Energies are in eV per $\mathrm{AO}_{2}$ unit cell.

fact that all the sesquioxides, $\mathrm{A}_{4} \mathrm{O}_{6}$, prefer the trivalent ground state configuration shows that the removal of $\mathrm{O}$ from the dioxide leads to localization of an additional $f$-electron. Whether the oxidation (Eq.(6)) or reduction (Eq. (8) ) process actually takes place depends on the thermodynamic conditions, in particular the $\mathrm{O}$ chemical potential.

The results for the calculated oxidation and reduction energies are presented in Fig. 6. A binding energy of $7.2 \mathrm{eV}$ has been adopted for the $\mathrm{O}_{2}$ molecule (relative to the non spin-polarized $\mathrm{O}$ atoms. The atomic spinpolarization energy [68] is $1.14 \mathrm{eV}$ per $\mathrm{O}$ atom). The accuracy of the energy differences is not expected to be very high due to the ASA and the neglect of structural optimizations. Note, however, that for $\mathrm{Pu}_{2} \mathrm{O}_{3}$, our calculated value of $2.6 \mathrm{eV} / \mathrm{PuO}_{2}$ for the reduction process, Eq. (9), lies within the range of $\mathrm{LDA}+\mathrm{U} / \mathrm{GGA}+\mathrm{U}(U=4$ $\mathrm{eV})$ values calculated by Jomard et al. 11], 2.59/2.05 $\mathrm{eV} / \mathrm{PuO}_{2}$, and Sun et al. 37], $1.50 / 2.00 \mathrm{eV} / \mathrm{PuO}_{2}$ (data points taken from their figure). Nevertheless, here we will only concentrate on trends, which come out quite clearly.

It emerges that $E^{o x}$ increases slightly and $E^{\text {red }}$ decreases as we move through the actinide series, which reflects the increasing f-electron binding energy. In Uranium an f-electron is readily promoted into the valence band to facilitate uptake of super-stoichiometric oxygen, while this becomes increasingly more difficult with heavier actinides. On the other hand the $\mathrm{U}$ f-manifold is very reluctant to take on an extra f-electron to form the $3+$ ion on reduction, while this becomes easier for the later actinides. The jump in reduction energy at Bk reflects the relative stability of the half-filled $f^{7}$ shell in $\mathrm{BkO}_{2}$.

According to the calculated energy differences, the only compound that oxidizes exothermically is $\mathrm{UO}_{2}$, whilst for all other actinide dioxides, the energy balance is positive and increasing with actinide number. This result is in overall good agreement with experimental data. $\mathrm{UO}_{2+x}$ has been synthesized up to $\mathrm{x}=0.25\left(\mathrm{U}_{4} \mathrm{O}_{9}\right)$. The crystal structure remains fluorite based, with the excess $\mathrm{O}$ atoms situated at interstitial sites. 69] With respect to $\mathrm{Np}$, the only known stable binary oxide, apart from $\mathrm{NpO}_{2}$, is $\mathrm{Np}_{2} \mathrm{O}_{5}$, with a structure that can no longer be described as underlying fluorite. [70] In particular, the fluorite derived $\mathrm{Np}_{4} \mathrm{O}_{9}$ does not seem to exist. With respect to $\mathrm{PuO}_{2+x}$, the present calculations confirm the stability of $\mathrm{PuO}_{2}$, in line with the majority of experimental investigations on this material. The same conclusion was reached in an earlier SIC-LSD study [43] where the possible discovery of a higher composition binary oxide, $\mathrm{PuO}_{2+x}(\mathrm{x} \leq 0.27)[71$ was discussed. Beyond $\mathrm{Pu}$ there have been no reports of super-stoichiometric oxides. The positive slightly increasing oxidation energies in Fig. 6 confirm that the dioxide for all the trans-plutonium actinides is increasingly stable against oxidation.

Concerning the reduction reaction in Eq. (8), it is found that the energy balance always favors the dioxide. With respect to the early actinides $\mathrm{U}$ and $\mathrm{Np}$ the large value of the reduction energy is in agreement with the experimental fact that neither $\mathrm{U}_{2} \mathrm{O}_{3}$ nor $\mathrm{Np}_{2} \mathrm{O}_{3}$ exists. The sesquioxides from $\mathrm{Pu}_{2} \mathrm{O}_{3}$ to $\mathrm{Cf}_{2} \mathrm{O}_{3}$ do exist, however they are according to our calculations in principle unstable in air towards further uptake of oxygen and formation of the dioxide. In practice there may be appreciable barriers to the actual transformation, 72] which furthermore may be influenced by thermodynamic conditions (temperature and pressure) not considered here.

\section{SUMMARIZING DISCUSSION}

From the study of the stable binary actinide oxides, a clear picture emerges that links the degree of oxidation to the degree of $f$-electron localization. In the early actinides, the $f$-electrons are less bound to the actinide ions which translates into valencies as high as $5+$ and $6+$ for U-oxides for example. As one progresses through the actinide series, the $f$-electrons become increasingly tightly bound to the actinide ion, and eventually for $\mathrm{Cf}$ only the $3+$ valency occurs naturally. The actinide ions play an active role in accomodating extra $\mathrm{O}$, as their localized $f$-electrons can act as electron reservoirs for the highly electronegative O-ion. In other words, whether the oxidation is favoured depends on the willingness of the actinide to delocalize further $f$-electrons. From the SIC-LSD study we find that the divalent configuration is never favoured, except maybe for EsO. Consequently, the monoxide never forms as the third electron is readily delocalized and made available for oxidation, which leads to the formation of trivalent sesquioxides. Whether the oxidation progresses further to produce the corresponding dioxide depends on delocalization of the 4th electron on the actinide ion. As we have seen this delocalization is less likely to happen for the late actinides, where the $f$-electrons tend to be more localized, and where experiment shows that the synthesis of $\mathrm{CfO}_{2}$ takes place under powerful oxidation conditions, e.g. in the presence 
TABLE IV: Groundstate valency configurations of the actinide and lanthanide oxides. Bold lettering indicates that the corresponding compound actually exists in nature.

\begin{tabular}{|c|c|c|c|c|c|c|c|c|}
\hline A & U & $\mathrm{Np}$ & $\mathrm{Pu}$ & $\mathrm{Am}$ & $\mathrm{Cm}$ & $\mathrm{Bk}$ & Cf & Es \\
\hline$\overline{\mathrm{AO}}$ & $5+$ & $4+$ & $3+$ & $3+$ & $3+$ & $3+$ & $3+$ & $3+/ 2+$ \\
\hline $\mathrm{A}_{2} \mathrm{O}_{3}$ & $4+$ & $4+$ & $3+$ & $3+$ & $3+$ & $3+$ & $3+$ & - \\
\hline $\mathrm{AO}_{2}$ & $5+/ 4+$ & $4+$ & $4+$ & $4+$ & $4+$ & $4+$ & $4+$ & - \\
\hline $\mathrm{R}$ & $\mathrm{Nd}$ & $\mathrm{Pm}$ & $\mathrm{Sm}$ & $\mathrm{Eu}$ & $\mathrm{Gd}$ & $\mathrm{Tb}$ & Dy & Ho \\
\hline $\mathrm{RO}$ & $3+$ & $3+$ & $3+/ 2+$ & $2+$ & $3+$ & $3+$ & $3+$ & $3+$ \\
\hline$\overline{\mathrm{R}_{2} \mathrm{O}_{3}}$ & $3+$ & $3+$ & $3+$ & $3+$ & $3+$ & $3+$ & $3+$ & $3+$ \\
\hline $\mathrm{RO}_{2}$ & $4+$ & $4+$ & $3+$ & $3+$ & $3+$ & $4+$ & $3+$ & $3+$ \\
\hline
\end{tabular}

of high pressure molecular or atomic oxygen. 72 For the early actinides, on the other hand, the oxidation to the dioxide occurs readily, and for example $\mathrm{U}_{2} \mathrm{O}_{3}$ and $\mathrm{Np}_{2} \mathrm{O}_{3}$ do not exist naturally. Finally, the further oxidation from dioxide to higher oxide only occurs for $\mathrm{UO}_{2}$, where both the $5+\left(\mathrm{U}_{2} \mathrm{O}_{5}\right)$ and $6+\left(\mathrm{UO}_{3}\right) \mathrm{U}$-valencies exist, and for $\mathrm{NpO}_{2}$ where the $5+$ valency is obtained in $\mathrm{Np}_{2} \mathrm{O}_{5}$. We can speculate here as to whether the fact that we actually determine a marginally pentavalent groundstate for $\mathrm{UO}_{2}$ (rather than the well established tetravalent configuration) is an indication that an additional electron $f$ electron is on the brink of delocalization, thus favouring the formation of higher U-oxides. The remaining dioxides are stable with respect to oxidation, as the gain in delocalizing the 5 th electron is no longer favorable compared to the corresponding loss in SIC energy.

In Table IV we have collected our results of the SICLSD total energy calculations for the actinide oxides (the upper 4 rows). The numbers indicate the ground state valency configurations that we have determined for a given compound. Bold large letters are used to indicate those compounds that actually do exist in nature. It clearly emerges that only in those cases where the calculated ground state valency agrees with the nominal charge expected from an ideal ionic picture, does the corresponding oxide seem to form. In other words, those oxides where our calculations predict a valency configuration that is not in agreement with simple charge counting will not form naturally, and the excess/shortage of charge indicates that oxidized/reduced compound will be favoured instead. These trends emphasize the very ionic nature of bonding in the actinide oxides.

It is interesting to compare these trends in oxidation of the actinides to the corresponding behaviour of the lanthanides. 73, 74] In the latter the $4 f$ electrons are overall more tightly bound to the lanthanide ion, which will favour lower oxidation numbers, compared to the spatially more extended $5 f$ electrons. The corresponding calculated valency configurations 73 are shown in Table IV (the lower 4 rows). The effect of the increased local- ization can be observed from the fact that a number of the lantanide monoxides actually exist, and it emerges from SIC-LSD studies that especially for the half-filled and filled shell $f$-electron systems, i.e. for $\mathrm{EuO}$ and $\mathrm{YbO}$, the divalent configuration is energetically most favorable. All the lanthanide sesquioxides occur in nature, and the ground state configuration of the corresponding lanthanide ions is trivalent. Even though the $4 f$-electrons tend to be more localized, the $3+$ valency occurs naturally, which is in agreement with the trivalent configuration being the most favourable for the lanthanide metals. With respect to further oxidation to the tetravalent dioxide, only $\mathrm{CeO}_{2}, \mathrm{PrO}_{2}$ (not shown in Table IV), and $\mathrm{TbO}_{2}$, are found to occur naturally or (for $\mathrm{TbO}_{2}$ ) under high $\mathrm{O}$ pressure, indicating that an additional $f$-electron only delocalizes in the very early lanthanides, i.e. in $\mathrm{Ce}$ and $\operatorname{Pr}$, where the $f$-electrons are less tightly bound, or in the middle of the series, where tetravalent $\mathrm{TbO}_{2}$ is favoured by the half-filled shell configuration. 73. Higher oxidation numbers than IV do not exist for the lanthanide oxides. Again we observe that agreement between nominal charge and calculated ground state valency is required for a given compound to exist.

\section{CONCLUSION}

We have studied the electronic structure of actinide oxides and specifically monoxides, sesquioxides and dioxides, within the ab initio SIC-LSD band structure method. By studying the oxidation and reduction reactions we have been able to conclude that the dioxides, from Np onwards, are the most stable compounds among the studied actinide oxides. Our study reveals a strong link between the preferred oxidation number and the degree of localization which is confirmed by comparing to the ground state configurations of the corresponding lanthanide oxides. The ionic nature of the actinide oxides is reflected in that only those compounds can form where the calculated ground state valency agrees with the nominal valency expected from a simple charge counting.

\section{Acknowledgment}

This research used resources of the Danish Center for Scientific Computing (DCSC) and of the National Energy Research Scientific Computing Center (NERSC). Research supported in part (GMS) by the Division of Materials Science and Engineering, Office of Basic Energy Science, U.S. Department of Energy. We gratefully acknowledge helpful discussions with M. S. S. Brooks.
[1] G. R. Choppin, J. O. Liljenzin, and J. Rydberg, in Radiochemistry and Nuclear Chemistry-3rd ed. (Butterworth-
Heinemann, 2001), chap. 21. 
[2] R. J. M. Konings, J. Nucl. Mater. 298, 255 (2001).

[3] M. Beauvy, T. Duverneix, C. Berlanga, R. Mazoyer, and C. Duriez, J. Alloys Compd. 271-273, 557 (1998).

[4] F. Weigel, J. J. Katz, and G. T. Seaborg, in The Chemistry of the Actinide Elements, edited by J. J. Katz, G. T. Seaborg, and L. R. Morss (Chapman Hall, New York, 1986), vol. 1, p. 680.

[5] C. Guéneau, C. Chatillon, and B. Sundman, J. Nucl. Mater. 378, 257 (2008).

[6] B. Johansson and H. Skriver, J. Magn. Magn. Mat. 29, 217 (1982).

[7] A. Svane, L. Petit, Z. Szotek, and W. M. Temmerman, Phys. Rev. B 76, 115116 (2007).

[8] T. Maehira and T. Hotta, J. Magn. Magn. Mater. 310, 754 (2007).

[9] J. C. Boettger and A. K. Ray, Int. J. Quantum Chem. 90, 1470 (2002).

[10] M. Freyss, N. Vergnet, and T. Petit, J. Nucl. Mater. 352, 144 (2006).

[11] G. Jomard, B. Amadon, F. Bottin, and M. Torrent, Phys. Rev B 78, 075125 (2008).

[12] V. I. Anisimov, J. Zaanen, and O. K. Andersen, Phys. Rev. B 44, 943 (1991).

[13] G. Kotliar and D. Vollhardt, Physics Today 57, 53 (2004).

[14] S. J. Savrasov, G. Kotliar, and E. Abrahams, Nature 410, 793 (2001).

[15] A. D. Becke, J. Chem. Phys. 98, 1372 (1993).

[16] J. P. Perdew and A. Zunger, Phys. Rev. B 23, 5048 (1981).

[17] D. Kasinathan, J. Kuneš, K. Koepernik, C. V. Diaconu, R. L. Martin, I. D. Prodan, G. E. Scuseria, N. Spaldin, L. Petit, T. C. Schulthess, et al., Phys. Rev. B 74, 195110 (2006).

[18] W. M. Temmerman, A. Svane, Z. Szotek, H. Winter, and S. V. Beiden, in Lecture Notes in Physics, edited by M. Dreyssé (Springer-Verlag, Berlin Heidelberg, 2000), vol. 535, p. 286.

[19] A. Svane, Phys. Rev. B 53, 4275 (1996).

[20] O. K. Andersen, Phys. Rev. B 12, 3060 (1975).

[21] G. C. Allen and P. M. Tucker, J. Chem. Soc. Dalton Trans. p. 470 (1973).

[22] W. P. Ellis, Surface Sci. 61, 37 (1976).

[23] D. J. Lam, J. B. Darby, and M. B. Newitt, in The Actinides, Electronic Structure and Related Properties, edited by A. J. Freeman and J. B. Darby (Academic, New York, 1974), vol. 11, p. 119.

[24] K. Winer, C. A. Colmenares, R. L. Smith, and F. Wooten, Surface Sci. 177, 484 (1986).

[25] M. Eckle and T. Gouder, J. Alloys Compd. 374, 261 (2004).

[26] K. Richter and C. Sari, J. Nucl. Mater. 148, 266 (1987).

[27] J. R. Naegele, L. E. Cox, and J. W. Ward, Inorganica Chimica Acta 139, 32 (1987).

[28] Y. Akimoto, J. Inorg. nucl. Chem. 29, 2650 (1967).

[29] J. Fahey, J. Peterson, and R. Baybarz, Inorg. Nucl. Chem. Letters 8, 101 (1972).

[30] P. Villars and L. D. Calvert, in Pearson's Handbook of Cystallographic Data for Intermetallic Phases, 2nd ed. (ASM International, Ohio, 1991).

[31] M. S. S. Brooks, J. Phys. F: Met. Phys. 14, 639 (1984).

[32] V. A. Gubanov, A. Rosén, and D. E. Ellis, J. Phys. Chem. Solids 40, 17 (1979).

[33] D. Courteix, J. Chayrouse, L. Heintz, and R. Baptist,
Solid State Commun. 39, 209 (1981).

[34] T. Gouder, P. M. Oppeneer, F. Huber, F. Wastin, and J. Rebizant, Phys. Rev. B 72, 115122 (2005).

[35] B. W. Veal, D. J. Lam, and H. R. Hoekstra, Phys. Rev. B 15, 2929 (1977).

[36] I. D. Prodan, G. E. Scuseria, and R. L. Martin, Phys. Rev. B 73, 045104 (2006).

[37] B. Sun, P. Zhang, and X. G. Zhao, J. Chem. Phys. 128, 084705 (2008).

[38] M. Wulff and G. H. Lander, J. Chem. Phys 89, 3295 (1988).

[39] J. C. Boettger and A. K. Ray, Int. J. Quantum Chem. 80, 824 (2000).

[40] Y. Yun, H. Kim, H. Kim, and K. Park, Nuclear Engineering and Technology 37, 293 (2005).

[41] S. L. Dudarev, G. A. Botton, S. Y. Savrasov, Z. Szotek, W. M. Temmerman, and A. P. Sutton, phys. stat. sol. (a) 166, 429 (1998).

[42] Q. Yin and S. Y. Savrasov, Phys. Rev. Lett. 100, 225504 (2008).

[43] L. Petit, A. Svane, Z. Szotek, and W. M. Temmerman, Science 301, 498 (2003).

[44] I. D. Prodan, G. E. Scuseria, and R. L. Martin, Phys. Rev. B 76, 033101 (2007).

[45] P. J. Kelly, M. S. S. Brooks, and R. Allen, J. Phys. C4, 184 (1979).

[46] P. J. Kelly and M. S. S. Brooks, J. Chem. Soc., Faraday Trans. 2 83, 1189 (1987).

[47] B. Johansson, J. Phys. Chem. Solids 39, 467 (1978).

[48] R. Troć and D. Kaczorowski, in Landolt-Bornstein: $\mathrm{Nu}$ merical Data and Functional Relationships in Science and Technology. New Series, Group III: Condensed Matter, Volume 27: Magnetic Properties of Non-Metallic Inorganic Compounds Based on Transition Elements, Subvolume C2: Binary Actinide Oxides, edited by H. J. Wijn (Springer, Berlin-Heidelberg, 1999).

[49] Y. Baer and J. Schoenes, Solid State Commun. 33, 885 (1980).

[50] J. M. Fournier and R. Troć, in Handbook on the Physics and Chemistry of the Actinides, edited by A. J. Freeman and G. H. Lander (North Holland, Amsterdam, 1985), vol. 2, chap. 2.

[51] J. Schoenes, J. Chem. Soc. Faraday Trans. 2 83, 1205 (1987).

[52] G. B. Ogden and C. T. Walker, Phys. Rev. Lett. 18, 11 (1967).

[53] K. H. Kang, H. J. Ryu, K. C. Song, and M. S. Yang, J. Nucl. Mater. 301, 242 (2002).

[54] C. Jorgensen, in Orbitals in Atoms and Molecules (Academic, London, 1962).

[55] L. J. Nugent, J. Inorg. Nucl. Chem. 32, 3485 (1970).

[56] S. Xia and J. C. Krupa, J. Alloys Compd. 307, 61 (2000).

[57] M. Idiri, T. L. Bihan, S. Heathman, and J. Rebizant, Phys. Rev B 70, 014113 (2004).

[58] J. P. Dancausse, R. G. Haire, S. Heathman, and U. Benedict, J. Nucl. Sci. Technol. Suppl. 3, 136 (2002).

[59] U. Benedict and C. Dufour, Physica B 102, 303 (1980).

[60] L. Soderholm, J. Less-Common Met. 133, 77 (1987).

[61] S. E. Nave, R. G. Haire, and P. G. Huray, Phys. Rev. B 28, 2317 (1983).

[62] J. R. Peterson and J. Fuger, J. Inorg. Nucl. Chem. 33, 4111 (1971).

[63] M. Noé and J. Fuger, Inorg. \& Nucl. Chem. Lett. 7, 421 (1971). 
[64] L. R. Morss, J. W. Richardson, C. W. Williams, G. H. Lander, A. C. Lawson, N. M. Edelstein, and G. V. Shalimoff, J. Less-Common Met. 156, 273 (1989).

[65] L. B. Asprey, F. H. Ellinger, S. Fried, and W. H. Zachariasen, J. Am. Chem. Soc. 77, 1707 (1955).

[66] M. Lüders, A. Ernst, M. Däne, Z. Szotek, A. Svane, D. Ködderitzsch, W. Hergert, B. L. Gyorffy, and W. M. Temmerman, Phys. Rev. B 71, 205109 (2005).

[67] I. D. Hughes, M. Däne, A. Ernst, W. Hergert, M. Lüders, J. P. Poulter, J. B. Staunton, A. Svane, Z. Szotek, and W. M. Temmerman, Nature 446, 650 (2007).

[68] E. L. P. Blanca, A. Svane, N. E. Christensen, C. O. Rodriguez, O. M. Cappannini, and M. S. Moreno, Phys. Rev B 48, 15712 (1993).

[69] G. C. Allen, P. M. Tucker, and J. W. Tyler, J. Phys.
Chem. 86, 224 (1982).

[70] T. Forbes, P. Burns, S. Skanthakumar, and L. Soderholm, J. Am. Chem. Soc. 129, 2760 (2007).

[71] J. M. Haschke, T. H. Allen, and L. A. Morales, Science 287, 285 (2000).

[72] R. D. Baybarz, R. G. Haire, and J. A. Fahey, J. Inorg. nucl. Chem. 34, 557 (1972).

[73] L. Petit, A. Svane, Z. Szotek, and W. M. Temmerman, Phys. Rev. B 72, 205118 (2005).

[74] L. Petit, A. Svane, Z. Szotek, and W. M. Temmerman, in Topics in Applied Physics, edited by M. Fanciulli and G. Scarel (Springer-Verlag, Berlin Heidelberg, 2007), vol. 106, p. 331. 